\title{
Development of a Doxycycline Hydrochloride-Loaded Electrospun Nanofibrous Membrane for GTR/GBR Applications
}

\author{
Lie-ni Jia, ${ }^{1}$ Xin Zhang, ${ }^{2}$ Hong-yu Xu, ${ }^{1}$ Fei Hua, ${ }^{3}$ Xiang-gang $\mathrm{Hu},{ }^{1}$ \\ Qiao Xie, ${ }^{1}$ Wei Wang, ${ }^{1}$ and Jun Jia ${ }^{1}$ \\ ${ }^{1}$ State Key Laboratory of Military Stomatology, Department of Prosthodontics, School of Stomatology, \\ The Fourth Military Medical University, Xian, Shaanxi 710032, China \\ ${ }^{2}$ Department of Stomatology, Central Hospital of Xi'an, Shaanxi 710032, China \\ ${ }^{3}$ Department of Pharmacy, Institute of Material Medical, The Fourth Military Medical University, Xian, Shaanxi 710032, China \\ Correspondence should be addressed to Jun Jia; jiajun@fmmu.edu.cn
}

Received 23 August 2015; Revised 2 January 2016; Accepted 11 February 2016

Academic Editor: Silvia Licoccia

Copyright (C) 2016 Lie-ni Jia et al. This is an open access article distributed under the Creative Commons Attribution License, which permits unrestricted use, distribution, and reproduction in any medium, provided the original work is properly cited.

\begin{abstract}
A drug-loaded membrane was prepared by electrospinning Poly $\varepsilon$-caprolactone (PCL) with doxycycline hydrochloride (DOX) (15$25 \% \mathrm{w} / \mathrm{w}$ ). Scanning electron microscopy (SEM) images revealed that fibrous average diameter decreased from $247.16 \pm 57.61 \mathrm{~nm}$ to $194.43 \pm 43.33 \mathrm{~nm}$ with the drug proportion increasing from $15 \%$ to $20 \% \mathrm{w} / \mathrm{w}$, while there was no significant difference between $20 \%$ and $25 \%$ groups. The polymer matrix showed good encapsulation value (58-75\%) for DOX, and the drug showed an amorphous manner in the polymer matrix. The agar diffusion test revealed that DOX-loaded membranes had an obvious inhibited effect on Aggregatibacter actinomycetemcomitans (Aa) and Porphyromonas gingivalis (Pg), respectively. In vitro release test showed that DOX could persistently be released for a prolonged time more than 28 days, and the DOX level in the eluent steadied at 3-5 $\mu \mathrm{g} / \mathrm{mL}$ which was all above the minimum inhibitory concentration (MIC) of DOX against Aa $(0.125 \mu \mathrm{g} / \mathrm{mL})$ and $\mathrm{Pg}(0.0625 \mu \mathrm{g} / \mathrm{mL})$. Cytocompatibility, assessed in human periodontal ligament cells (hPLCs) by MTT-test and the morphology of cells on the surface of DOX-loaded membranes by SEM, indicated that all of the investigated nanofibrous membranes can be used to treat periodontal disease by integrating the GTR/GBR operation and antibiotic therapy. Above all, DOX-loaded nanofibrous membranes could have a persistent inhibited effect on periodontal pathogens to provide a relatively sterile environment for tissue repair and regeneration.
\end{abstract}

\section{Introduction}

Periodontal disease is a group of chronic inflammatory diseases of periodontium, which can be highly aggressive and lead to the destruction of periodontium, eventually resulting in tooth loss. Meanwhile, the inflammation caused by bacterial plaque infection has been thought the dominant cause for periodontal damage and the important factor affecting the clinical outcome of periodontitis treatment. During the past decades, some approaches have been used to treat this disease, which usually have two integral parts: surgical debridement and antibiotic therapy.

The primary method to treat periodontal disease is cleaning bacterial plaque top to bottom through surgical debridement, including flap debridement and/or flap curettage, or through mechanical elimination by patients themselves. Moreover, two surgical approaches for regeneration, guide tissue regeneration (GTR) and guide bone regeneration (GBR), have been increasingly used for the repair of damaged periodontal tissue [1-3]. Although surgical debridement, mechanical elimination, and tissue regeneration are the utmost important approaches for periodontitis treatment, the oral environment is filled with microorganisms, and the bacterial plaque could continuously form on the teeth, which may have a negative effect on the surgical treatment outcome.

Antibiotic therapy as an assistant method plays the irreplaceable role in periodontitis treatment. Compared with widespread systemic administration, which frequently generates excessive doses leading to substantial local and possible 
undesirable side effects or favoring the development of resistances, local delivery of antibiotics is a more beneficial choice for periodontitis treatment [4]. For example, directed antibiotic delivery was utilized to treat osteomyelitis, a prolonged inflammation of bone, which causes destruction of bone tissue and vascular channels due to pathogenic microorganism $[5,6]$.

In terms of periodontitis treatment, a drug-loaded biodegradable GTR/GBR membrane can be even more promising in meeting our goals, providing a relatively sterile environment to promote the damaged tissue regeneration. On the one hand, drug-loaded GTR/GBR membranes could place a barrier over the denuded root surface and the debrided periodontal defect to exclude epithelial growth and further to allow specifically periodontal ligament and alveolar bone cells to repopulate the isolate space [7-9]. On the other hand, it has the capacity to eliminate microorganism infection and inhibit the bacterial colonization on the root surface [10].

One popular method to fabricate such drug-loaded biodegradable membranes is electrospinning, a promising and versatile processing technique which utilizes electrical forces to produce nanofibers using polymer solutions [11]. The resulting fibers have diameters in the range of several hundred nanometers to a few microns, large surface area to volume ratio, high porosity, and three-dimensional network structure which can possibly enhance the adhesion, proliferation, and growth of cells [12]. Moreover, drug can be capsulated directly into electrospun fibers by electrospinning a blended solution of drug and polymer, and the fibers can be used as carriers in controlled drug release $[13,14]$. Electrospun membranes can be cut into any size and shapes, making them suitable for various types of clinical applications [15, 16]. Meanwhile, electrospun nanofibrous membranes have a structure mimicking native extracellular matrix (ECM). They can provide both chemical and physical cues to modulate cell adhesion and differentiation and to promote tissue regeneration [17]. All of these features make electrospun nanofibrous membranes widely used in the biomedical field.

Poly $\varepsilon$-caprolactone (PCL), as a semicrystalline biodegradable aliphatic polyester, has many features, such as slow biodegradability, good biocompatibility, high drug permeability, and better mechanical property for resisting the mechanical stress caused by the surgical operation and the tissue of operation zone, which make it a good candidate material for biomedical applications [16]. Another advantage of PCL is that it does not produce a local acidic environment as it degrades $[16,18]$ which could lead to an acidosis and have negative effect on wound healing [19]. Therefore, PCL was chosen to fabricate the membranes and serve as the drug carrier in this study.

Doxycycline hydrochloride (DOX) is one of the most common broad spectrum antibiotics. It is actively against most of the periodontal pathogens due to the low minimum inhibitory concentration (MIC) [20], has the ability of collagenase inhibition, anti-inflammatory action, and inhibition of bone absorption, and could bind to the hard tissue walls of pockets to establish a drug reservoir [21]. Previous research had showed that DOX with concomitant mechanical instrumentation and scaling and root planning (SRP) had a useful effect on periodontitis treatment over 9-month study period [20]. Moreover, some researches have evaluated effectiveness of doxycycline nanofibers in the treatment of chronic periodontitis as an adjunct to SRP, by putting drug-loaded nanofibers into periodontal pocket. The results showed that the group treated with SRP and DOX-loaded nanofibers was significantly better than group treated with SRP alone [21].

Different with drug delivery nanofibers, the drug-loaded GTR/GBR membrane, being implanted into periodontal tissue, should meet more stringent requirements. On the one hand, drug-loaded GTR/GBR membrane should have good biocompatibility and affinity with periodontal tissue to promote repair and regeneration. On the other hand, the medicated GTR/GBR membrane should have the capacity to eliminate microorganism infection and inhibit the bacterial colonization on the root surface, which is the foundation for tissue repair and regeneration.

The aim of present study was to produce nanofibrous membranes containing the antibiotic DOX by electrospinning technique, which have potential to treat periodontal disease by integrating the GTR/GBR operation and antibiotic therapy. The surface morphology, drug entrapment efficiency, drug release profile, cytocompatibility, and bioactivity of these membranes were studied.

\section{Materials and Methods}

2.1. Materials. Poly $\varepsilon$-caprolactone (PCL, molecular weight $=$ $80,000 \mathrm{~g} / \mathrm{mol}$, inherent viscosity $0.81 \mathrm{dL} / \mathrm{g}$ in $\mathrm{CHCl}_{3}$ ) was purchased from Sigma, USA. 1,1,1,3,3,3-Hexafluoro-2-propanol (HFIP) (Aladdin, China) was selected as the solvent for electrospinning solution. Doxycycline hydrochloride (DOX) (Xiya Reagent, China) has a purity of $98 \%$. 3-(4,5Dimethylthiazol-2-yl-2,5-diphenyltetrazolium bromide) (MTT) and antibiotic antimycotic solution (AAS) were purchased from Genview, USA. Dulbecco's modified eagle medium (DMEM) and fetal bovine serum were purchased from Hyclone, USA. Mueller-Hinton Broth (MH broth) and Schaedler Agar were purchased from Oxoid, UK. All the chemicals used were of analytical grade.

2.2. Electrospinning. The $10 \%(\mathrm{w} / \mathrm{w})$ electrospinning solution was prepared by dissolving an appropriate amount of PCL and DOX in HFIP. The DOX proportion was in the range of $15-25 \%(w / w)$ with respect to PCL. Magnetic stirring was applied for at least $12 \mathrm{~h}$ at room temperature to obtain homogeneous solution prior to electrospinning. Then, the solution was transferred to a $5 \mathrm{~mL}$ syringe with a needle $(21 \mathrm{G}$, internal diameter of $0.5 \mathrm{~mm}$ ) which was connected to the high-voltage power supply. The fibers were collected on an aluminum-foil coated on aluminum plate connected to a negative voltage. Electrospinning was carried out under ambient conditions $\left(25^{\circ} \mathrm{C}\right.$ and relative humidity $\left.40 \%\right)$. The electrical potential used was $18 \mathrm{KV}$, and the feed rate of the polymer solution was maintained at $1 \mathrm{~mL} / \mathrm{h}$ using a syringe pump; the distance between the needle tip and the aluminum plate was $15 \mathrm{~cm}$. A series of fibers with different drug load (15\%,20\%, and 25\% $\mathrm{w} / \mathrm{w}$ ) were prepared by electrospinning 5 hours without an interval, respectively [18]. 
2.3. Scanning Electron Microscopy. The morphology of drugloaded fibers was characterized using a scanning electron microscope (SEM, s-4800, HITACHI, Japan). Before examination, samples were gold sputter-coated under argon using a Denton Vacuum Desk II sputter coater (Moorestown, NJ) at $50 \mathrm{~m}$ Torr to render them electrically conductive. SEM images were recorded at an excitation voltage of $5 \mathrm{kV}$. The nanofibrous average diameter was determined by measuring the diameter of fibers $(n=100)$ from SEM images using the Image-Pro Plus 6 software (National Institutes of Health, USA).

2.4. Differential Scanning Calorimetry. The nature of DOX in the nanofibers was assessed by performing differential scanning calorimetry (DSC) on crude PCL granules, pure PCL nanofibers, pure DOX powders, $15 \%$ (w/w) DOX-loaded nanofibers, 20\% (w/w) DOX-loaded nanofibers, and 25\% (w/w) DOX-loaded nanofibers. DSC analysis was carried out using a DSC Q2000 calorimeter (TA instruments, New Castle, USA). Sealed samples were put into the aluminum pan and heated at $10^{\circ} \mathrm{C} / \mathrm{min}$ from $50^{\circ} \mathrm{C}$ to $300^{\circ} \mathrm{C}$ under a $50 \mathrm{~mL} /$ min flow of nitrogen and subsequently cooled to $50^{\circ} \mathrm{C}$ at $10^{\circ} \mathrm{C} / \mathrm{min}$.

2.5. Entrapment Efficiency. Entrapment efficiency was determined by rinsing a known mass of sample with phosphate buffer solution ( $\mathrm{PBS}, 10 \mathrm{mmol} / \mathrm{liter}, \mathrm{pH}=7.4$ ), then dissolving the rinsed sample in HFIP, and monitoring the UV absorbance at $\lambda=349 \mathrm{~nm}$ (the molar extinction coefficient is $1.02 \times 10^{5} \mathrm{~mol} / \mathrm{L} \cdot \mathrm{cm}^{-1}$ ). The amount of DOX was obtained from the calibration curve of DOX in the same solution. The entrapment efficiency was calculated by the following equation [18]:

Entrapment efficiency (\%)

$$
\begin{aligned}
= & \frac{\text { weight of drug in the membrane }}{\text { theoretical weight of drug loaded in the membrane }} \\
& \times 100 \% \text {. }
\end{aligned}
$$

2.6. In Vitro Release Behavior. In vitro elution method was used to investigate the release characteristics of DOX from the nanofibrous membranes. The medicated electrospun nanofibrous membranes were cut into pieces with a dimension of $2.5 \mathrm{~cm} \times 2.5 \mathrm{~cm}$ (about $250-350 \mu \mathrm{m}$ in thickness). The samples were accurately weighed and rinsed by $5 \mathrm{~mL}$ PBS $(10 \mathrm{mmol} / \mathrm{liter}, \mathrm{pH}=7.4)$, then the rinsed membranes were placed into $5 \mathrm{~mL}$ of PBS and incubated in a constant-temperature shaker (CHA-S, Guohua, China) at $37^{\circ} \mathrm{C}, 150 \mathrm{rpm}$. At predetermined time interval, eluents of $150 \mu \mathrm{L}$ for each sample were withdrawn for UV analyses, and the remaining medium was replaced with fresh PBS to maintain sink conditions. The amount of released DOX was determined using a Multimode Reader equipped with a UV-Vis detector (Infinite $200 \mathrm{PRO}$, TECAN, USA). The UV absorbance of DOX in buffer solution was determined at $\lambda=349 \mathrm{~nm}$ (the molar extinction coefficient is $1.09 \times$ $10^{5} \mathrm{~mol} / \mathrm{L} \cdot \mathrm{cm}^{-1}$ ) and converted to the DOX concentration according to the calibration curve of the DOX in the same buffer. The results were recorded as an average of three measurements [22].

\subsection{Cytocompatibility of Antibiotic-Loaded PCL Nanofibers}

2.7.1. MTT-Test. The proliferation of cells on drug-loaded nanofibers was measured to evaluate the cytocompatibility of the membranes. The samples were incubated with human periodontal ligament cells (hPDLCs) which were cultivated in DMEM with $10 \%$ of fetal calf serum and $0.1 \%$ of AAS. The cytocompatibility of the samples was examined by MTT assay. Electrospun drug-loaded membranes (with the diameter of $5 \mathrm{~mm}$ and the thickness of $250-350 \mu \mathrm{m}$ ) were sterilized by $\mathrm{Co}^{60}$ irradiation and rinsed by PBS. The rinsed membranes were incubated in 96-well tissue culture plates with hPDLCs $\left(5 \times 10^{3}\right.$ cells/well) at $37^{\circ} \mathrm{C}$ under $5 \% \mathrm{CO}_{2}-95 \%$ air conditions. Cell proliferation was assessed at $1,3,5$, and 7 days by MTT assay. Pure PCL membranes were used as a control group [7].

2.7.2. Cell Morphology on Drug-Loaded Membranes Study. The investigation of cell morphology on drug-loaded membranes was carried out by SEM. Sterilized membranes ( $8 \mathrm{~mm} \times 8 \mathrm{~mm}, 250-350 \mu \mathrm{m})$ were incubated in 24-well culture plates with hPDLCs $\left(1 \times 10^{5}\right.$ cells/well $)$ at $37^{\circ} \mathrm{C}$ under $5 \%$ $\mathrm{CO}_{2}-95 \%$ air conditions. After $24 \mathrm{~h}$, membranes were rinsed twice by PBS and fixed with $2.5 \%$ glutaraldehyde overnight at $4^{\circ} \mathrm{C}$, rinsed twice with PBS for $15 \mathrm{~min}$ each, and dehydrated using the gradient concentration of ethanol (50\%, 70\%, 80\%, $90 \%$, and $95 \%$ ) and then pure ethanol twice for $15 \mathrm{~min}$ each. Finally, the membranes were left air-dried overnight and analyzed by SEM to investigate the morphology of attached cells on them [23].

2.8. Bioactivity of Antibiotic-Loaded Nanofibers. The agar diffusion test, which has been considered as the standard method to evaluate antibiotic susceptibility, was used to demonstrate the antimicrobial effect of antibiotic-containing electrospun matrices on periodontopathogens. For this study two pathogenic periodontal bacterial strains were used: Aggregatibacter actinomycetemcomitans (Aa) and Porphyromonas gingivalis $(\mathrm{Pg})$. Each bacterial strain was cultivated in sterile $\mathrm{MH}$ broth enriched with vitamin $\mathrm{K}$ overnight at $37^{\circ} \mathrm{C}$ under anaerobic conditions. The inoculum was diluted to $\sim 10^{6} \mathrm{CFU} / \mathrm{mL}$. $100 \mu \mathrm{L}$ of each bacterial suspension was spread onto a Petri dish with Schaedler Agar enriched with 1\% of vitamin $\mathrm{K}$ and $10 \%$ of sheep blood. Sterilized $5 \mathrm{~mm}$ roundshaped samples $(250-350 \mu \mathrm{m}$ in thickness) were placed on blood agar plates containing bacterial lawns of Pg and Aa, respectively. 48 hours after anaerobic incubation, the zone of inhibition (in $\mathrm{mm}$ ) was measured. Five specimens were tested for each group [24].

The MICs of DOX against Aa and Pg were determined by an antibiotic tube diluted method in $\mathrm{MH}$ broth. DOX was diluted serially 2 -fold in tubes containing $0.5 \mathrm{~mL}$ of the $\mathrm{MH}$ broth [7].

2.9. Statistical Analysis. Results were shown as the mean values and their standard deviations (SD). One-way analysis of variance (ANOVAs) followed by Tuky's multiple 


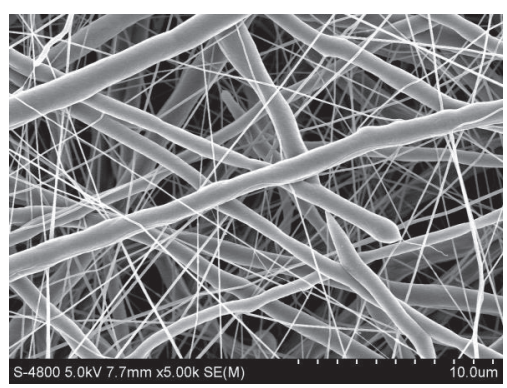

(a)
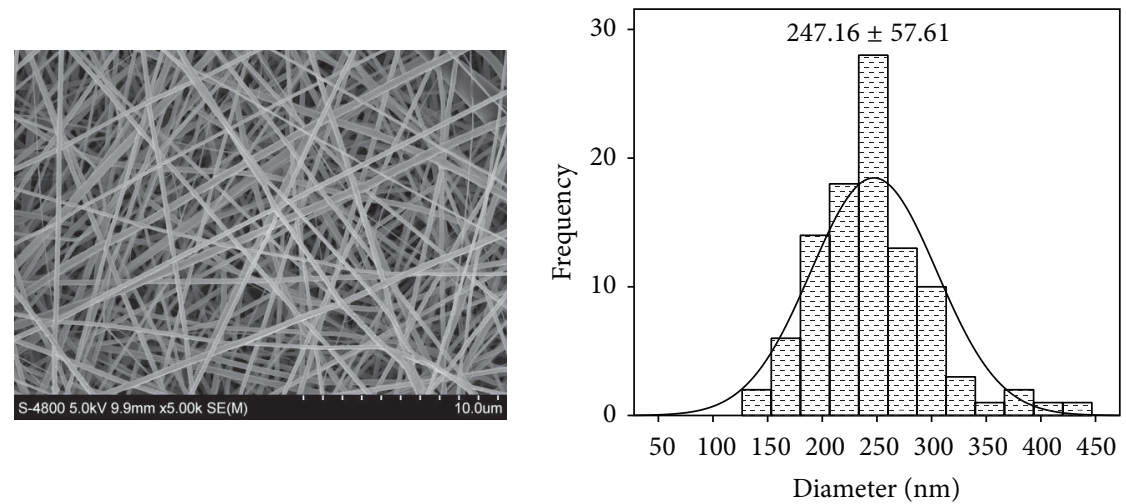

(b)
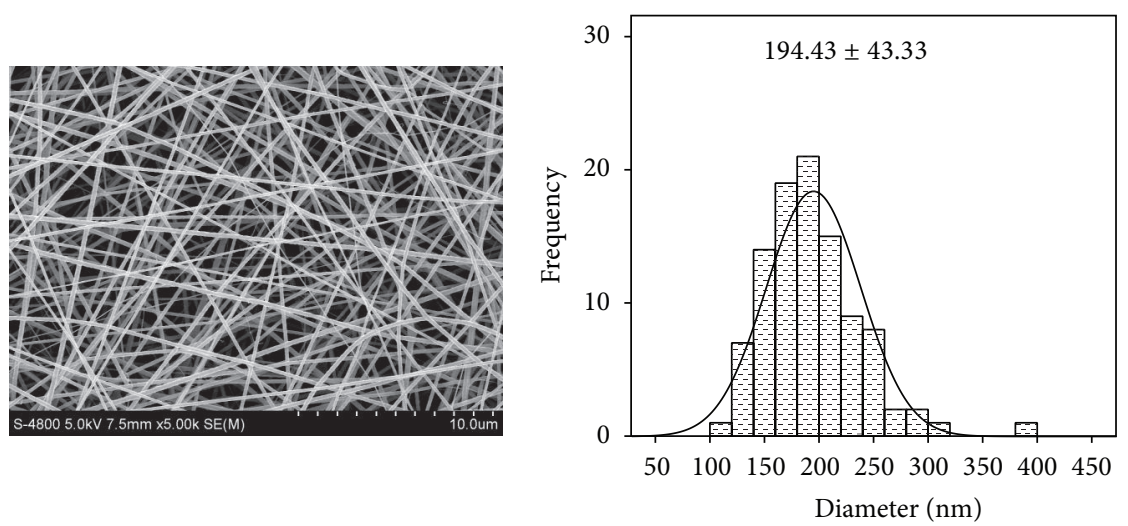

(c)
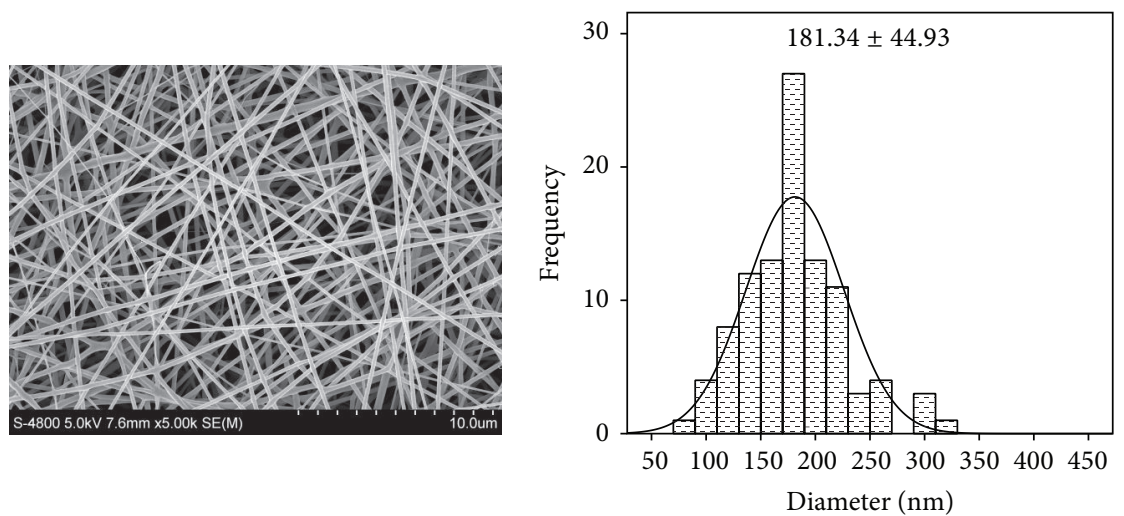

(d)

Figure 1: (a-d) Representative scanning electron micrographs of total 10\% (w/w) PCL and DOX solution. (a) Pure PCL nanofibers; (b) 15\% (w/w) DOX-loaded nanofibers; (c) 20\% (w/w) DOX-loaded PCL nanofibers; (d) 25\% (w/w) DOX-loaded PCL nanofibers.

comparisons was used to compare the groups for differences in fiber diameter, cell viability, and the zone of inhibition. The comparisons for entrapment efficiency were performed by Kruskal-Wallis $H$ tests. A 5\% significance level was used for all tests and all the analyses were conducted using SPSS statistical software (IBM; version 19).

\section{Results}

3.1. Scanning Electron Microscope. HFIP is a good solvent for both PCL and DOX, while desired nanofibers could not be obtained by using a $10 \%(\mathrm{w} / \mathrm{w})$ PCL solution in HFIP without the addition of DOX. The amount of DOX added to the solution had a significant effect on the morphology and diameter of electrospun nanofibers, as shown in Figures 1(a)1(d). The SEM photographs demonstrated that all electrospun drug-loaded nanofibers produced in this study were smooth and beadless. By increasing the drug percentage from $15 \%$ to $20 \%(\mathrm{w} / \mathrm{w}$ ), average diameter of drug-loaded electrospun nanofibers decreased from $247.16 \pm 57.61 \mathrm{~nm}$ to $194.43 \pm$ $43.33 \mathrm{~nm}(p<0.01)$. However, there was no significant difference between $25 \%(w / w)(181.34 \pm 44.93 \mathrm{~nm})$ and $20 \%(w / w)$ groups $(p=0.144)$. 
TABLE 1: Entrapment efficiency of different groups after rinsing $(n=$ $3)$.

\begin{tabular}{lcc}
\hline Group & $\begin{array}{c}\text { Theoretical } \\
\text { drug loading } \\
(\% \text { w/w })\end{array}$ & Entrapment efficiency (\%) \\
\hline A & 15 & $58.23 \pm 4.31$ \\
B & 20 & $72.87 \pm 3.67$ \\
C & 25 & $75.14 \pm 4.25$ \\
\hline
\end{tabular}

Entrapment efficiency of different groups: (A) refers to $15 \%$ w/w DOX-loaded PCL nanofibers; (B) refers to 20\% w/w DOX-loaded PCL nanofibers; (C) refers to $25 \% \mathrm{w} / \mathrm{w}$ DOX-loaded PCL nanofibers.

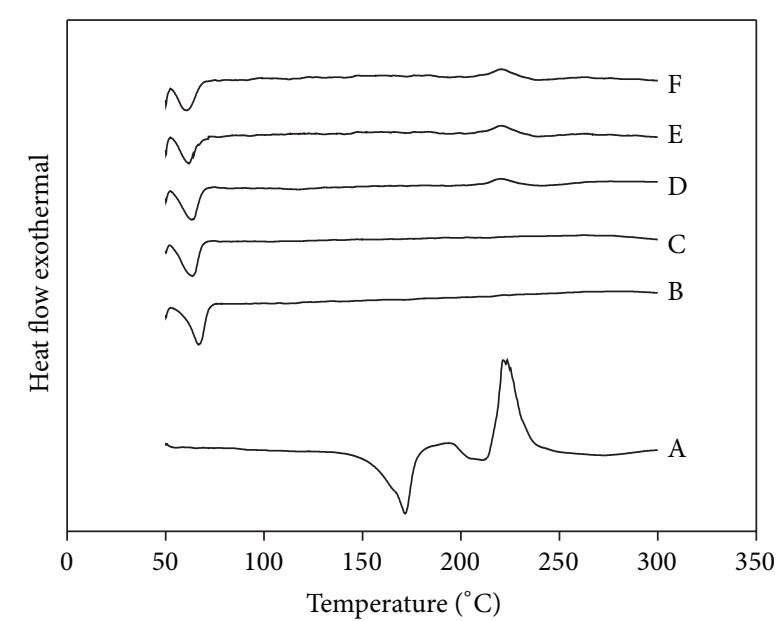

FIGURE 2: DSC thermograms of (A) pure doxycycline; (B) pure PCL granules; (C) pure PCL nanofibers; (D) 15\% (w/w) DOX-loaded nanofibers; (E) 20\% (w/w) DOX-loaded nanofibers; and (F) 25\% $(\mathrm{w} / \mathrm{w})$ DOX-loaded nanofibers.

3.2. Differential Scanning Calorimetric Study. DSC data are presented in Figure 2. The thermogram curve of DOX powders showed an endothermic sharp peak at $171.5^{\circ} \mathrm{C}$ due to melting temperature of semicrystalline DOX, and it showed carbonation reaction at $223.5^{\circ} \mathrm{C}$. Also, an endothermic melting peak at $66^{\circ} \mathrm{C}$ was observed for crude PCL granules and pure PCL nanofibers, while the melting endotherm peak of DOX was not detected in drug-loaded nanofibers. The absence of detectable crystalline domain showed that DOX was molecularly dispersed in amorphous state in polymeric matrix. Meanwhile, with DOX added to the solutions, the melting enthalpy of PCL nanofibers became lower than crude PCL granules and pure PCL nanofibers and appeared at almost the same temperature of $60-63^{\circ} \mathrm{C}$. With the increase of DOX proportion in nanofibers, the melting enthalpy of PCL nanofibers decreased.

3.3. Entrapment Efficiency. With the increase of drug proportion, the entrapment efficiency of polymer nanofibers for DOX from $15 \%(\mathrm{w} / \mathrm{w})$ to $25 \%(\mathrm{w} / \mathrm{w})$ increased from $58.23 \%$ value to $75.14 \%$ value, as Table 1 showed ( $p=0.0218)$.

3.4. In Vitro Release Behavior. The results of in vitro cumulative release of DOX from drug-containing electrospun

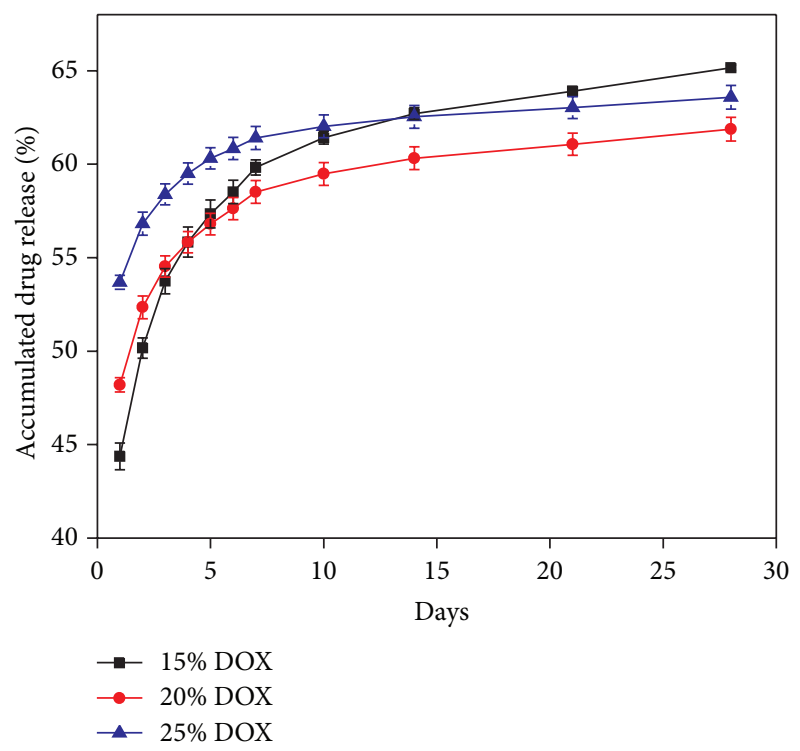

FIGURE 3: Drug release profiles of PCL nanofibers loaded with different percentages of DOX: 15\% DOX refers to $15 \%(w / w)$ DOXloaded nanofibers; 20\% DOX refers to $20 \%$ (w/w) DOX-loaded nanofibers; 25\% DOX refers to 25\% (w/w) DOX-loaded nanofibers.

membranes are shown in Figure 3. A fast initial release phase was observed during the first day. Within the first day, $44.37 \%, 48.20 \%$, and $53.68 \%$ of the total DOX were released from different groups drug-loaded nanofibers (15\% $\mathrm{w} / \mathrm{w}, 20 \% \mathrm{w} / \mathrm{w}$, and $25 \% \mathrm{w} / \mathrm{w}$ ), respectively. The DOX release was more rapid during the first five days, and there was a more linear release of it after that period. DOX level in the eluent remained steady at $3-5 \mu \mathrm{g} / \mathrm{mL}$ and the drug could be persistently released more than 28 days. Comparing the drug release curves of three groups, fiber loaded $15 \%(w / w)$ DOX released drug more rapidly than the fiber loaded $20 \%(\mathrm{w} / \mathrm{w})$ DOX and $25 \%(\mathrm{w} / \mathrm{w})$ one, and there was the same situation for $20 \%(\mathrm{w} / \mathrm{w})$ and $25 \%(\mathrm{w} / \mathrm{w})$ groups.

\subsection{Cytocompatibility of Antibiotic-Loaded PCL Nanofibers}

3.5.1. MTT-Test. The results of MTT assays are presented in Figure 4. The pure PCL membranes, and drug-loaded membranes with different DOX proportion were compared with the blank control. After culture in vitro, the optical density value decreased for drug-loaded membranes compared with blank control and pure PCL groups. Moreover, with the increase of DOX proportion, the optical density value decreased, while the optical density value increased with the extension of incubation time from 1 day to 7 days for the same proportion of DOX-loaded group. All medicated membranes analyzed in this part presented a difference results on cell proliferation compared with the control group.

3.5.2. Cell Morphology on Drug-Loaded Membranes Study. Figures 5(a)-5(i) show the morphology of hPLDCs on the surface of different DOX-loaded nanofibers after incubation for $24 \mathrm{~h}$ by SEM. The imagines clearly exhibited that fusiform 


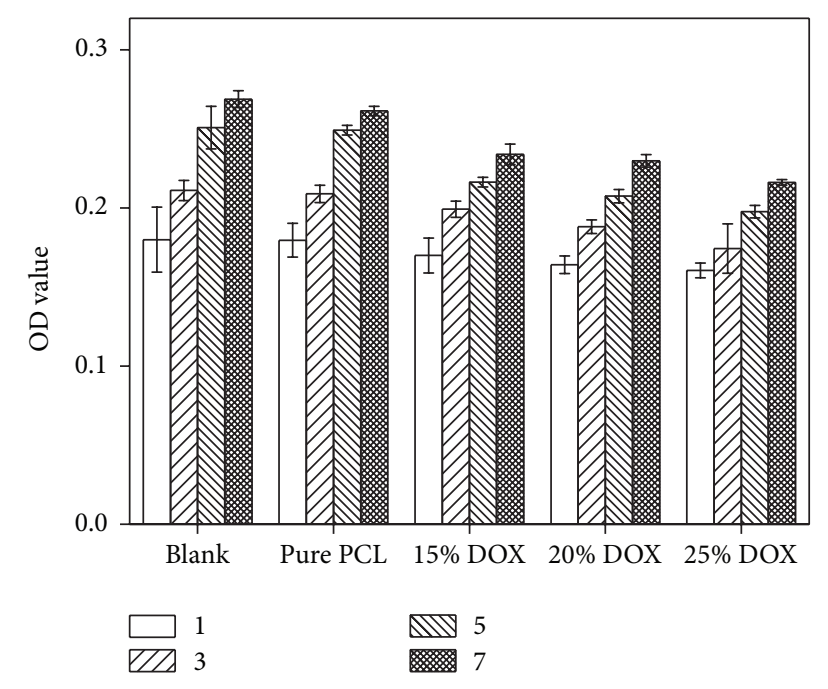

FIgURE 4: The optical density (OD) value of cytotoxicity test results from MTT assays of cell viability. The numbers 1, 3, 5, and 7 refer to the days for cell culture, respectively.

or polygon-shaped hPLDCs fully spread out and interconnected with each other on the surface of the drug-loaded membranes, and its dendritic protrusions closely connected with fibers. Cells grew along the fibrous surface through pseudopodia. This clearly suggested that cells had a better adhesion on the DOX-loaded nanofibrous scaffolds and expressed their characteristic morphology, which implied the cytocompatibility of the drug-loaded membranes [23].

3.6. Bioactivity of Antibiotic-Loaded Nanofibers. The results of agar diffusion test revealed that DOX-loaded nanofiber could inhibit the growth of both $\mathrm{Aa}$ and $\mathrm{Pg}$, as shown in Figure 6. The average inhibition zone diameter of $15 \%(\mathrm{w} / \mathrm{w})$ group was $26.03 \mathrm{~mm}$ against $\mathrm{Aa}$ and $26.01 \mathrm{~mm}$ against $\mathrm{Pg}$. The $20 \%(\mathrm{w} / \mathrm{w}$ ) group was $28.15 \mathrm{~mm}$ against $\mathrm{Aa}$ and $29.65 \mathrm{~mm}$ against Pg. The 25\% (w/w) group was $28.73 \mathrm{~mm}$ against Aa and $31.48 \mathrm{~mm}$ against Pg. As the results indicated, there was a clearly dose-dependent inhibition for Aa and Pg. With the same $15 \%(w / w)$ of DOX, no difference was found between the two bacterial strains ( $p=0.953$ ), while the effect of DOX against Pg was better than against Aa for growth inhibition, with the same $20 \%(\mathrm{w} / \mathrm{w})(p=0.004)$ and $25 \%(\mathrm{w} / \mathrm{w})(p<$ 0.001 ) proportions.

The results of antibiotic tube diluted method showed that the MIC of DOX against Aa was $0.125 \mu \mathrm{g} / \mathrm{mL}$ and against $\mathrm{Pg}$ was $0.0625 \mu \mathrm{g} / \mathrm{mL}$.

\section{Discussion}

Traditional GTR/GBR membranes can only serve as physical barrier to prevent the growth of connective and epithelial tissue and cannot effectively inhibit the colonization of periodontal pathogen on the surface of root and periodontal tissue, which may compromise the repair and regeneration of periodontal tissues [25]. Compared with traditional GTR/ GBR membranes, the antibiotic-loaded membranes, made by electrospinning from biodegradable polymer, have several advantages. First, antibiotic-loaded membranes could provide appropriate concentration of antibiotics for killing bacteria and inhibiting the bacterial colonization for a prolonged time. Second, the biodegradable membranes could eliminate the need for surgical removal. Last, because the membranes dissolve slowly, the bone defect zone will also slowly be filled with tissue [26].

In current research, we successfully prepared DOXloaded nanofibrous membrane by electrospinning technique, while pure PCL solution could not acquire desired nanofibers without the addition of DOX in the same electrospinning conditions, as shown in Figures 1(a)-1(d). And, there was a reverse relationship between the concentration of DOX and the nanofibrous average diameter from $20 \%(\mathrm{w} / \mathrm{w})$ to $15 \%$ $(\mathrm{w} / \mathrm{w})$. Generally, cation and anion, dissociated by ionogenic substance, could significantly change the charge density and improve the electrical conductivity of the solution [27]. With the increased DOX concentration, the conductivity of the electrospinning solution increased and more electric charges forces were imposed on the jet under the electrical field. Moreover, with the increased solution conductivity, bending instability could be increased during the electrospinning. So, the jet path became more elongated and more stretched. Both higher elongation forces and greater bending instability resulted in fibers with smaller diameter [18]. And, compared with DOX-loaded groups, pure 10\% (w/w) PCL solution had a relatively lower conductivity, which made the jet could not elongate and stretch enough in the electrical field and presented the morphology as Figure 1(a) showed. However, increasing solution's conductivity by adding DOX had another opposite effect, accelerating spinning droplet to move in the electric field, which could increase electrospun nanofibrous diameter [28]. This could explain why there was no difference between the average diameter of PCL with $20 \%(\mathrm{w} / \mathrm{w})$ DOX and $25 \%(\mathrm{w} / \mathrm{w})$ groups.

DOX has a hydrophilic property, and it is easier to dissolve in water than in organic solvent. Lack of solubility in the polymer solution may cause a poor dispersion of the drug inside the solution. During electrospinning of such drug dispersions, the drug molecules might migrate on or near the fibrous surface, resulting in a subsequent burst release [29]. In this research, successful encapsulation of drug into the nanofibers was the main concern of ideally producing drugloaded GTR/GBR membranes and prolonged drug release. HFIP, chosen to be the cosolution for both polymer PCL and drug, has a good solubility for DOX. As the DSC results showed, the absence of detectable crystalline domain indicated that DOX was molecularly dispersed in amorphous state in polymeric matrix. With the increasing of DOX in nanofibers, the melting enthalpy of PCL nanofibers decreased which indicated that increasing the amount of DOX in the semicrystalline PCL nanofibers caused a reduction in the crystallinity.

The results of entrapment efficiency of different formulations after rinsing displayed distinctively different entrapment efficiency to the amount of incorporated drug. Increasing the amount of DOX led to further exposure of drug to the nanofibrous surface, which can dissolve in the rinsing water 


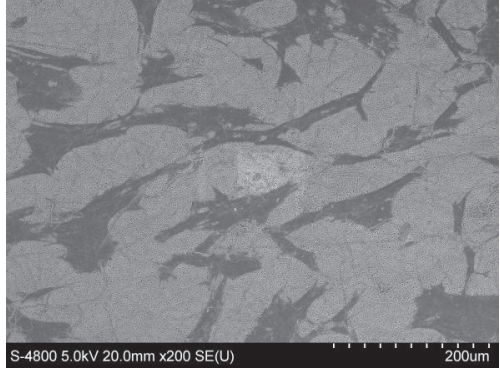

(a)

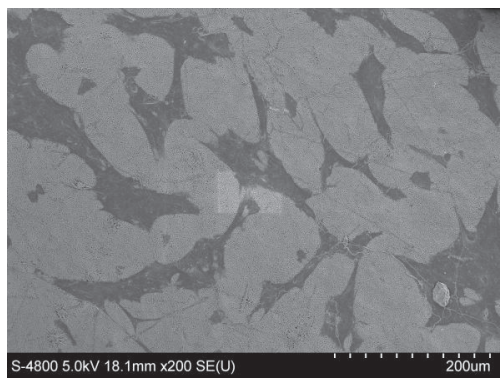

(d)

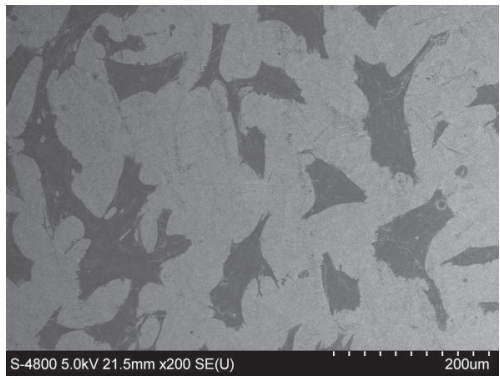

(g)

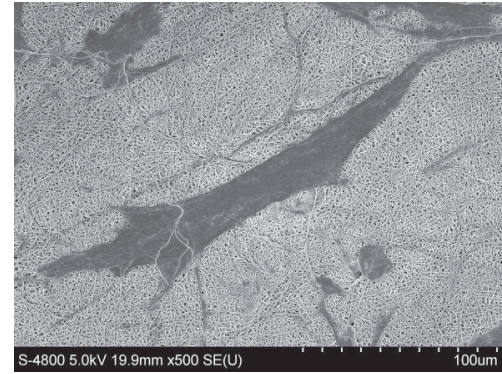

(b)

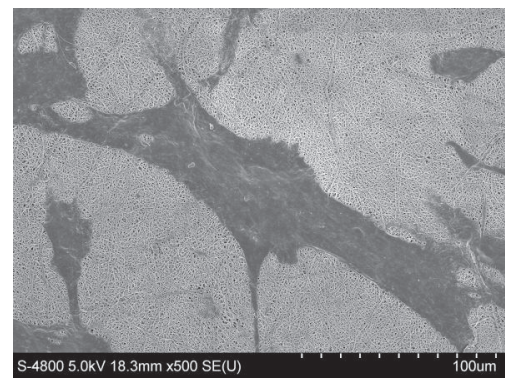

(e)

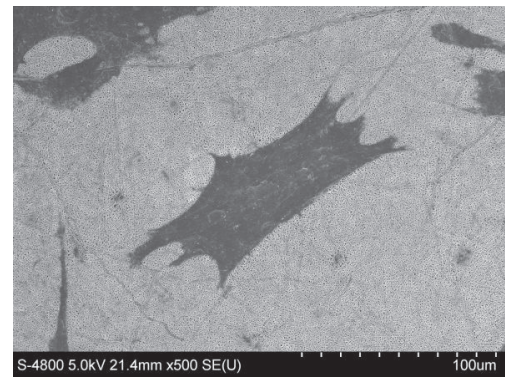

(h)

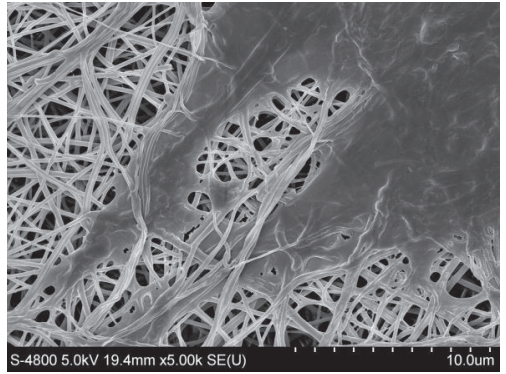

(c)

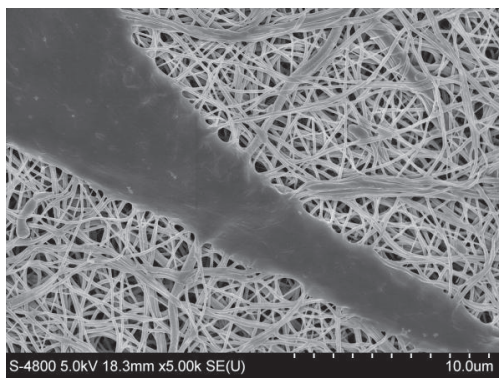

(f)

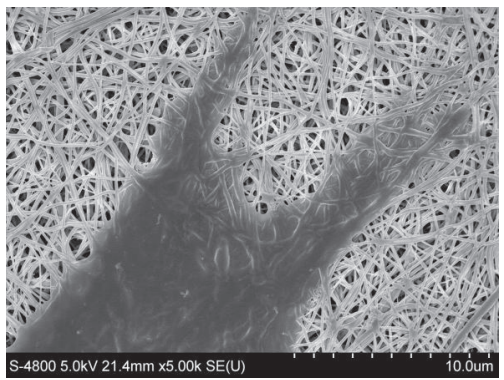

(i)

FIGURE 5: Morphology of hPLDCs on drug-loaded membrane with different concentration DOX after cultivation for 24 hours: (a), (b), and (c) are the group of $15 \% \mathrm{w} / \mathrm{w}$ DOX-loaded membranes at 200x, 500x, and 5000x magnification; (d), (e), and (f) are the group of 20\% w/w DOX-loaded membranes at 200x, 500x, and 5000x magnification; (g), (h), and (i) are the group of 25\% w/w DOX-loaded membrane at 200x, 500x, and 5000x magnification.

and be washed out easily. This could explain why entrapment efficiency did not increase with the drug proportion improvement.

Though it is believed that the solved drug in the polymer solution has a higher tendency to migrate to the surface or near the surface of nanofibers during the electrospinning process at a higher drug concentration, there is a concentration limitation [18]. When drug around the nanofibrous surface reaches a certain concentration limit, it tends to distribute into the fibers and be entrapped. That could be said that, by improving the drug-loaded concentration, the amount of drug on the nanofibrous surface increased, while the proportion of drug in the surface to the total drug-loaded membrane was decreased. Meanwhile, as mentioned in DSC test, with increasing DOX concentration in PCL, the crystallinity of the polymer carrier reduced. Drug may be entrapped into the amorphous regions of semicrystalline polymer, from where drug release occurs firstly [16]. Therefore, the exposure and diffusion of DOX to the PBS became higher, which resulted in a fast drug release rate for all the three groups of different concentration drug-loaded nanofibers at the first five days, and the amount of initial drug release had a positive correlation with the drug load. But, after the first initial release, all groups showed a more linear release profile of DOX which may attribute to drug release by diffusion or permeation of the inner drug through PCL matrix and the degradation of polymeric carrier. When eluent's concentration improved, it can limit the drug diffusion. This may result in little relationship with the membranes quality in this research, the eluent concentration remained steady at $3-5 \mu \mathrm{g} / \mathrm{mL}$, and higher quality of DOX load had a relatively lower accumulated drug release ratio. While the daily concentration obtained was completely dependent on the weight (or size) of the membranes and the volume of the eluent fluid, the $2.5 \mathrm{~cm} \times 2.5 \mathrm{~cm}$ membranes in $5 \mathrm{~mL}$ PBS may not be a suitable biologic test. The accumulated drug release should be a more valid calculation [7].

Moreover, the rate of drug release could be modulated by changing the morphology, porosity, and composition of 


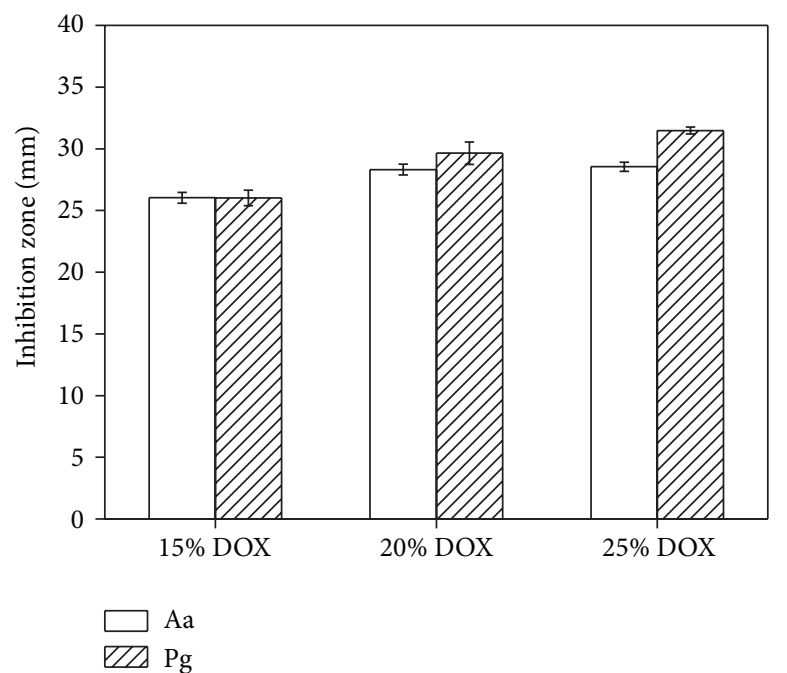

(a)

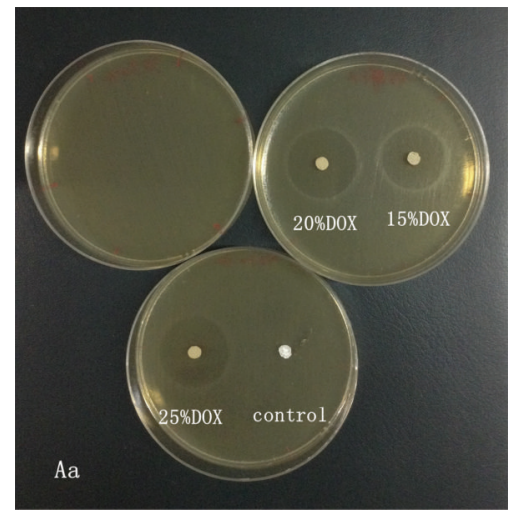

(b)

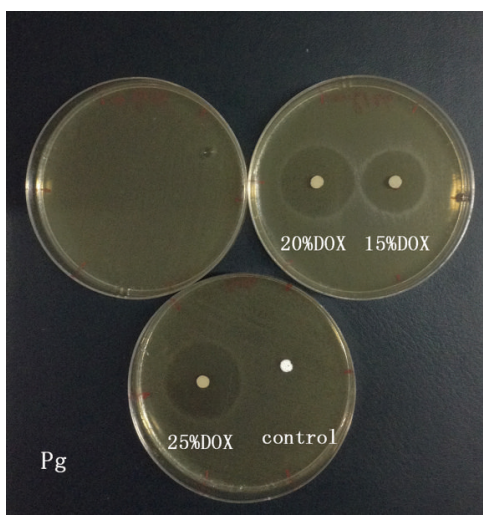

(c)

FIGURE 6: (a-c) Growth inhibition of Aa and Pg following 48 hours. (a) The analysis of inhibition zone diameter for drug-loaded membranes against $\mathrm{Aa}$ and $\mathrm{Pg}$; (b-c) representative macrophotographs showing growth inhibition of Aa and Pg, respectively.

nanofibers [30]. Very small diameter of nanofibers can provide a short diffusion passage length [31] and their high surface area to volume ratio also is helpful to mass transfer and efficient drug release [32]. For this research, the diameter of PCL nanofiber with $15 \%$ w/w DOX was larger than the $20 \%$ and $25 \%$ groups, but it also had a wider distribution. So the surface area of membrane may have no difference among the three groups. This could explain why the results of this research were not consistent with the regular phenomenon that lower diameters may have a higher drug release rate, and fiber with different average diameter had no significant effect on drug release rate in this test.

Electrospinning is a technology that can architecturally (in terms of geometry, morphology, or topography) and biochemically fabricate engineered cellular scaffolds that mimic the native extracellular matrix (ECM). Cells sense chemical and physical signals from ECM and scaffolding material both directly and indirectly via interfering protein adsorption [12]. MTT-test is a method to investigate the proliferation of cells. As shown in Figure 4, there was a difference for cell proliferation between the DOX-loaded and control groups.
One possible reason for the relatively low cell proliferation in DOX-loaded groups is that the sudden drug release may induce a high drug concentration which may impact cell adhesion, proliferation, and differentiation. But the physical and chemical properties of the membrane surface (roughness, wettability, surface charge property and porosity, etc.) also add another variable [33]. The exact reasons for difference in MTT-test results also need a further study. Meanwhile, cells were constantly proliferating during the period of culture from 1 day to 7 days in all groups. It indicated that the electrospun DOX-loaded nanofibers can be used to treat periodontal disease by integrating the GTR/GBR operation and antibiotic therapy, although cell proliferation in DOXloaded groups was lower than the control groups in this study.

In current research, antibacterial property of the DOXloaded membranes was evaluated using agar diffusion assay. Drug-loaded mats were tested on the growth inhibition against two main bacteria strains involved in periodontal infections, $\mathrm{Aa}$ and $\mathrm{Pg}$. The experimental results showed that DOX-loaded membranes could effectively inhibit the growth of periodontal pathogens (Figures $6(\mathrm{a})-6(\mathrm{c})$ ). What is more, 
the MIC of DOX against Aa was $0.125 \mu \mathrm{g} / \mathrm{mL}$ and against Pg was $0.0625 \mu \mathrm{g} / \mathrm{mL}$, and the DOX concentration of eluent during the vitro drug release test was above them. From what has been discussed above, electrospun DOX-loaded nanofibers have a consistent inhibited effect on periodontal pathogens to provide a relatively sterile environment for tissue repairmen and regeneration.

\section{Conclusion}

GTR/GBR membranes have been widely used in the surgical therapy of periodontal disease to regenerate lost periodontium. In this research, we successfully prepared DOX-loaded membranes by electrospinning for GTR/GBR investigation. Drug in electrospinning solution could be well entrapped by PCL matrix and had a sustained release to inhibit the of colonization periodontal pathogen over a long period of time. The structure and composition of the DOX-loaded electrospun nanofibrous membranes in this research provide a valuable bioactive addition to traditional GTR/GBR membranes for periodontitis treatment to eliminate microorganism infection and inhibit the bacterial colonization. However, about 40$50 \% \mathrm{w} / \mathrm{w}$ of total drug release from drug-loaded membranes occurred in the early drug release process, which might lead to a high local drug concentration not needed. So the next aim of our work is to reduce the drug proportion in the early release and further improve its cell affinity by changing the drug-loaded structure and physical and chemical properties of the membranes, to provide a more desirable antibioticloaded membrane for the investigation of periodontal tissue regeneration.

\section{Disclosure}

Xin Zhang is co-first author.

\section{Competing Interests}

The authors declare no competing interests to this work.

\section{Acknowledgments}

The authors would like to acknowledge the financial support by the National Science Foundation of China (no. 81270036). This investigation was supported by School of Stomatology, Institute of Material Medical School of Pharmacy, and Department of Military Toxicology, the Fourth Military Medical University.

\section{References}

[1] M. C. Bottino, V. Thomas, and G. M. Janowski, "A novel spatially designed and functionally graded electrospun membrane for periodontal regeneration," Acta Biomaterialia, vol. 7, no. 1, pp. 216-224, 2011.

[2] E. Milella, P. A. Ramires, E. Brescia, G. La Sala, L. Di Paola, and V. Bruno, "Physicochemical, mechanical, and biological properties of commercial membranes for GTR," Journal of Biomedical Materials Research, vol. 58, no. 4, pp. 427-435, 2001.
[3] J. Behring, R. Junker, X. F. Walboomers, B. Chessnut, and J. A. Jansen, "Toward guided tissue and bone regeneration: morphology, attachment, proliferation, and migration of cells cultured on collagen barrier membranes. A systematic review," Odontology, vol. 96, no. 1, pp. 1-11, 2008.

[4] G. Greenstein and A. Polson, "The role of local drug delivery in the management of periodontal diseases: a comprehensive review," Journal of Periodontology, vol. 69, no. 5, pp. 507-520, 1998.

[5] K. Garvin and C. Feschuk, "Polylactide-polyglycolide antibiotic implants," Clinical Orthopaedics and Related Research, no. 437, pp. 105-110, 2005.

[6] L. Lazzarini, J. T. Mader, and J. H. Calhoun, "Osteomyelitis in long bones," Journal of Bone and Joint Surgery A, vol. 86, no. 10, pp. 2305-2318, 2004.

[7] D. W.-C. Chen, F.-Y. Lee, J.-Y. Liao, S.-J. Liu, C.-Y. Hsiao, and J.-K. Chen, "Preclinical experiments on the release behavior of biodegradable nanofibrous multipharmaceutical membranes in a model of four-wall intrabony defect," Antimicrobial Agents and Chemotherapy, vol. 57, no. 1, pp. 9-14, 2013.

[8] S. Nyman, J. Gottlow, T. Karring, and J. Lindhe, "The regenerative potential of the periodontal ligament. An experimental study in the monkey," Journal of Clinical Periodontology, vol. 9, no. 3, pp. 257-265, 1982.

[9] J. Gottlow, S. Nyman, T. Karring, and J. Lindhe, "New attachment formation as the result of controlled tissue regeneration," Journal of Clinical Periodontology, vol. 11, no. 8, pp. 494-503, 1984.

[10] S. E. Gilchrist, D. Lange, K. Letchford, H. Bach, L. Fazli, and H. M. Burt, "Fusidic acid and rifampicin co-loaded PLGA nanofibers for the prevention of orthopedic implant associated infections," Journal of Controlled Release, vol. 170, no. 1, pp. 6473, 2013.

[11] N. Bhardwaj and S. C. Kundu, "Electrospinning: a fascinating fiber fabrication technique," Biotechnology Advances, vol. 28, no. 3, pp. 325-347, 2010.

[12] T. J. Sill and H. A. von Recum, "Electrospinning: applications in drug delivery and tissue engineering," Biomaterials, vol. 29, no. 13, pp. 1989-2006, 2008.

[13] S. G. Kumbar, L. S. Nair, S. Bhattacharyya, and C. T. Laurencin, "Polymeric nanofibers as novel carriers for the delivery of therapeutic molecules," Journal of Nanoscience and Nanotechnology, vol. 6, no. 9-10, pp. 2591-2607, 2006.

[14] Y. J. Son, W. J. Kim, and H. S. Yoo, "Therapeutic applications of electrospun nanofibers for drug delivery systems," Archives of Pharmacal Research, vol. 37, no. 1, pp. 69-78, 2014.

[15] M. Zamani, M. P. Prabhakaran, and S. Ramakrishna, "Advances in drug delivery via electrospun and electrosprayed nanomaterials," International Journal of Nanomedicine, vol. 8, pp. 29973017, 2013.

[16] E. Luong-Van, L. Grøndahl, K. N. Chua, K. W. Leong, V. Nurcombe, and S. M. Cool, "Controlled release of heparin from poly( $\varepsilon$-caprolactone) electrospun fibers," Biomaterials, vol. 27, no. 9, pp. 2042-2050, 2006.

[17] J. Chen, B. Zhou, Q. Li et al., "PLLA-PEG-TCH-labeled bioactive molecule nanofibers for tissue engineering.", International journal of nanomedicine, vol. 6, pp. 2533-2542, 2011.

[18] M. Zamani, M. Morshed, J. Varshosaz, and M. Jannesari, "Controlled release of metronidazole benzoate from poly epsiloncaprolactone electrospun nanofibers for periodontal diseases," European Journal of Pharmaceutics and Biopharmaceutics, vol. 75 , no. 2, pp. 179-185, 2010. 
[19] M. Reise, R. Wyrwa, U. Müller et al., "Release of metronidazole from electrospun poly(L-lactide-co-D/L-lactide) fibers for local periodontitis treatment," Dental Materials, vol. 28, no. 2, pp. 179-188, 2012.

[20] S. Garrett, D. F. Adams, G. Bogle et al., "The effect of locally delivered controlled-release doxycycline or scaling and root planing on periodontal maintenance patients over 9 months," Journal of Periodontology, vol. 71, no. 1, pp. 22-30, 2000.

[21] T. P. Chaturvedi, R. Srivastava, A. K. Srivastava, V. Gupta, and P. K. Verma, "Doxycycline poly e-caprolactone nanofibers in patients with chronic periodontitis-a clinical evaluation," Journal of Clinical and Diagnostic Research, vol. 7, no. 10, pp. 2339-2342, 2013.

[22] M. Jannesari, J. Varshosaz, M. Morshed, and M. Zamani, "Composite poly(vinyl alcohol)/poly(vinyl acetate) electrospun nanofibrous mats as a novel wound dressing matrix for controlled release of drugs," International Journal of Nanomedicine, vol. 6, pp. 993-1003, 2011.

[23] S. Gautam, A. K. Dinda, and N. C. Mishra, "Fabrication and characterization of PCL/gelatin composite nanofibrous scaffold for tissue engineering applications by electrospinning method," Materials Science and Engineering C, vol. 33, no. 3, pp. 12281235, 2013.

[24] M. C. Bottino, R. A. Arthur, R. A. Waeiss, K. Kamocki, K. S. Gregson, and R. L. Gregory, "Biodegradable nanofibrous drug delivery systems: effects of metronidazole and ciprofloxacin on periodontopathogens and commensal oral bacteria," Clinical Oral Investigations, vol. 18, no. 9, pp. 2151-2158, 2014.

[25] I. Brook, "Microbiology and management of periodontal infections," General Dentistry, vol. 51, no. 5, pp. 424-428, 2003.

[26] G. Polimeni, J. M. Albandar, and U. M. E. Wikesjö, "Prognostic factors for alveolar regeneration: effect of space provision," Journal of Clinical Periodontology, vol. 32, no. 9, pp. 951-954, 2005.

[27] H. Fong, I. Chun, and D. H. Reneker, "Beaded nanofibers formed during electrospinning," Polymer, vol. 40, no. 16, pp. 4585-4592, 1999.

[28] P. Supaphol, C. Mit-Uppatham, and M. Nithitanakul, "Ultrafine electrospun polyamide- 6 fibers: effect of emitting electrode polarity on morphology and average fiber diameter," Journal of Polymer Science B: Polymer Physics, vol. 43, no. 24, pp. 36993712, 2005.

[29] J. Zeng, L. Yang, Q. Liang et al., "Influence of the drug compatibility with polymer solution on the release kinetics of electrospun fiber formulation," Journal of Controlled Release, vol. 105, no. 1-2, pp. 43-51, 2005.

[30] K. Kim, Y. K. Luu, C. Chang et al., "Incorporation and controlled release of a hydrophilic antibiotic using poly(lactideco-glycolide)-based electrospun nanofibrous scaffolds," Journal of Controlled Release, vol. 98, no. 1, pp. 47-56, 2004.

[31] Z.-M. Huang, C.-L. He, A. Yang et al., "Encapsulating drugs in biodegradable ultrafine fibers through co-axial electrospinning," Journal of Biomedical Materials Research A, vol. 77, no. 1, pp. 169-179, 2006.

[32] G. Verreck, I. Chun, J. Rosenblatt et al., "Incorporation of drugs in an amorphous state into electrospun nanofibers composed of a water-insoluble, nonbiodegradable polymer," Journal of Controlled Release, vol. 92, no. 3, pp. 349-360, 2003.

[33] M. Şimşek, M. Çapkin, A. Karakeçili, and M. Gümüşderelioğlu, "Chitosan and polycaprolactone membranes patterned via electrospinning: effect of underlying chemistry and pattern characteristics on epithelial/fibroblastic cell behavior," Journal of Biomedical Materials Research Part: A, vol. 100, no. 12, pp. 3332-3343, 2012. 

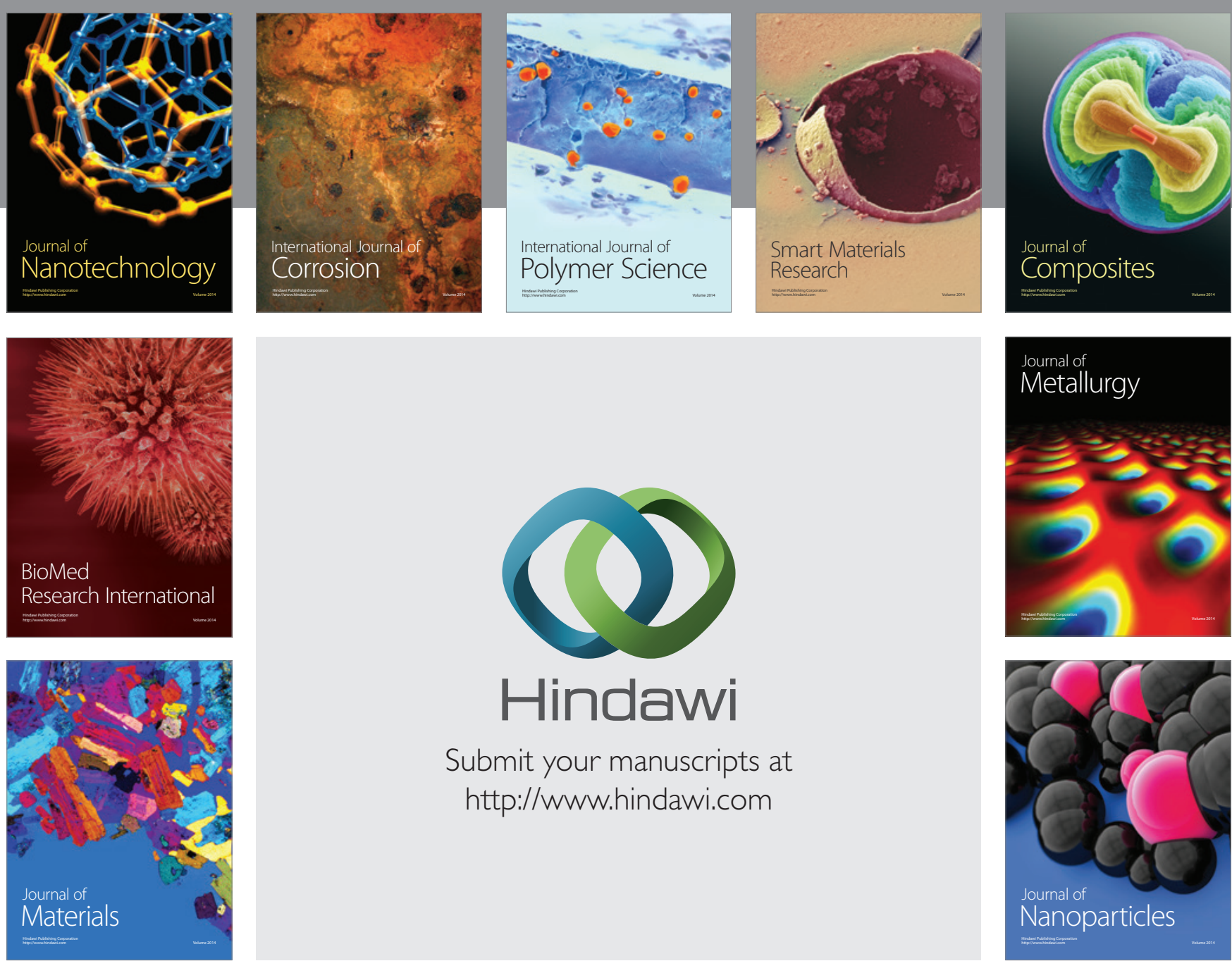

\section{Hindawi}

Submit your manuscripts at

http://www.hindawi.com

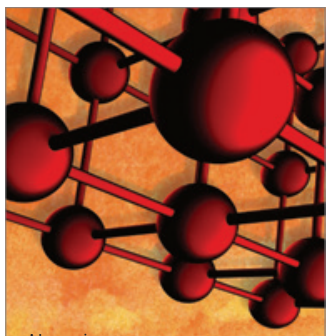

Materials Science and Engineering
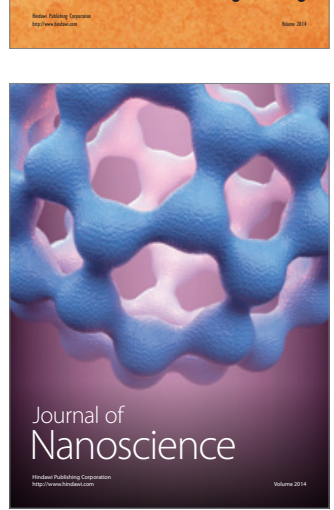
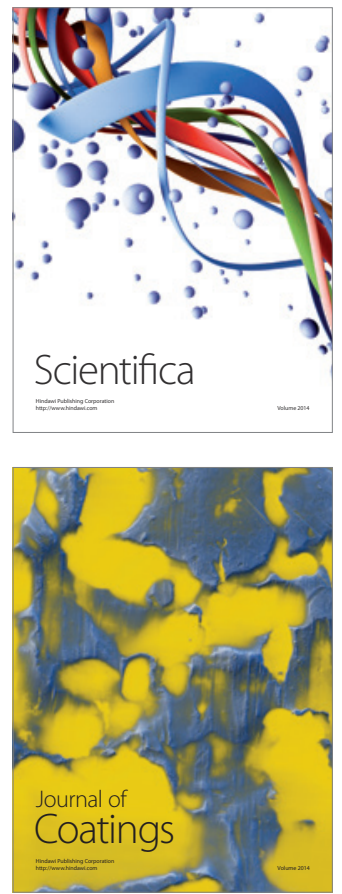
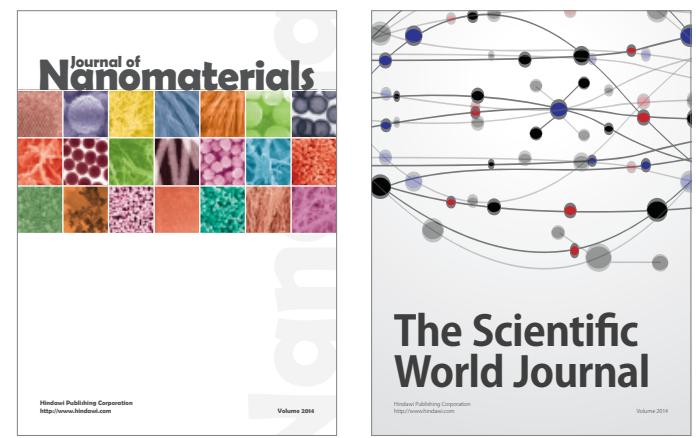

The Scientific World Journal
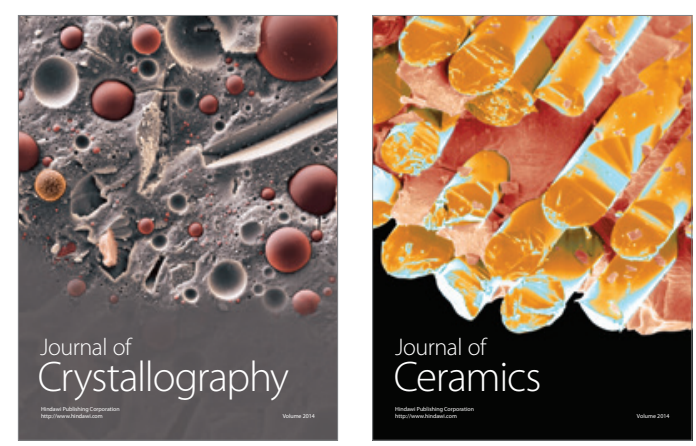
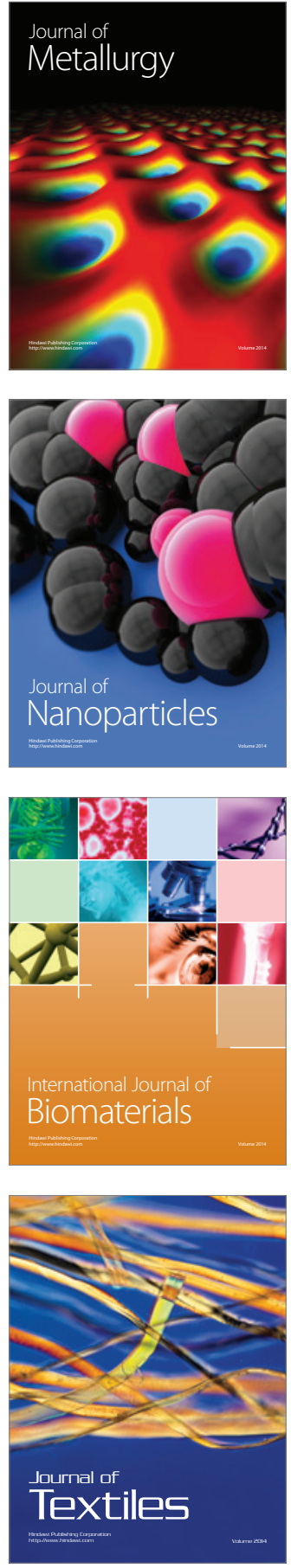